
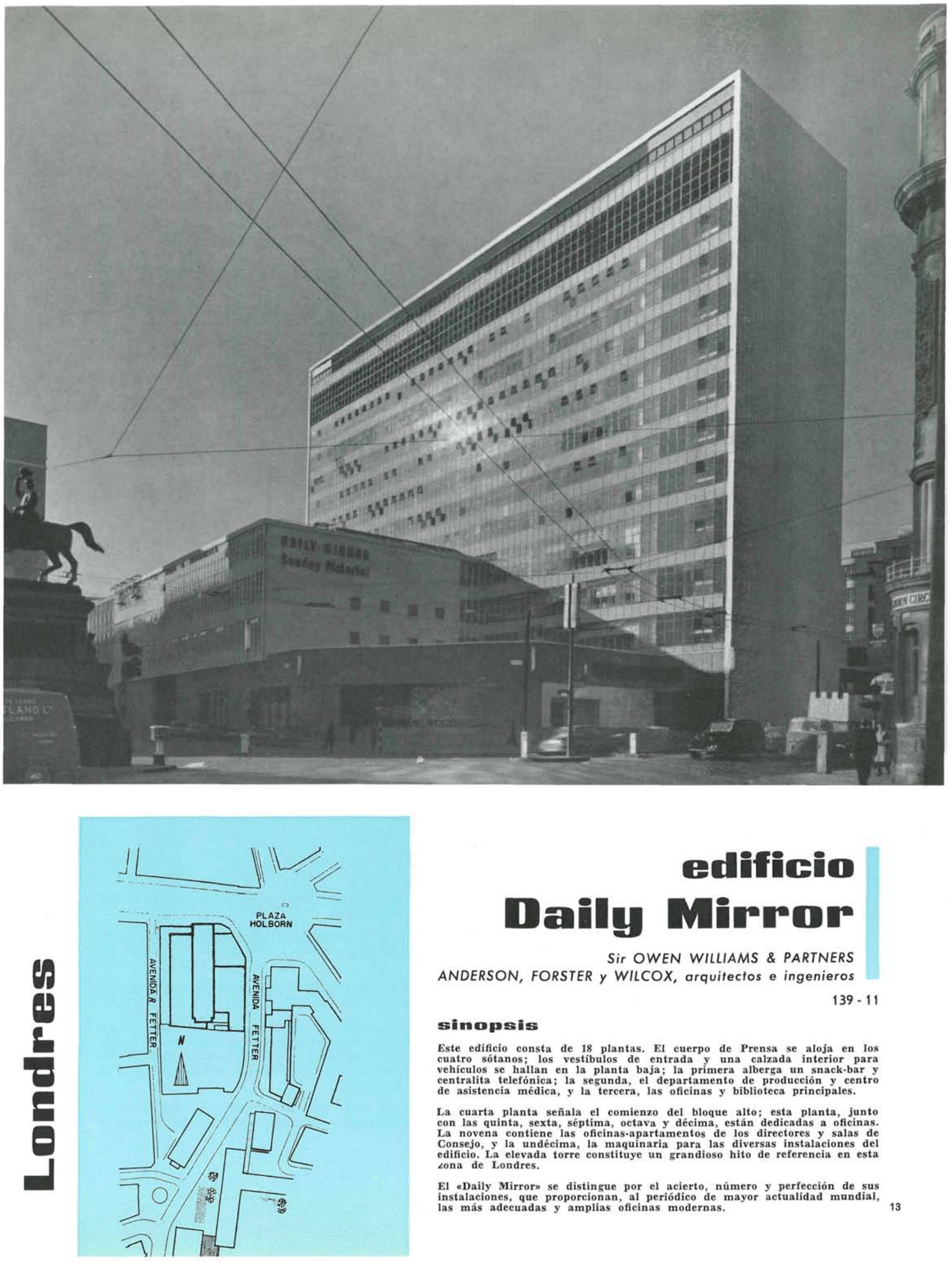

\title{
edificio Daily Mirror
}

Sir OWEN WILLIAMS \& PARTNERS ANDERSON, FORSTER y WILCOX, arquitectos e ingenieros

$139-11$

\section{sing opsis}

Este edificio consta de 18 plantas. El cuerpo de Prensa se aloja en los cuatro sótanos; los vestibulos de entrada y una calzada interior para vehículos se hallan en la planta baja; la primera alberga un snack-bar y centralita telefonica; la segunda, el departamento de producción y centro la tercera, las oficinas y biblioteca principales.

La cuarta planta señala el comienzo del bloque alto; esta planta, junto con las quinta, sexta, séptima, octava y décima, están dedicadas a oficinas. La novena contiene las oficinas-apartamentos de los directores y salas de edificio. La elevada torre constituye un grandioso hito de referencia en esta Lona de Londres.

El $\propto$ Daily Mirrorn se distingue por el acierto, número y perfección de sus instalaciones, que proporcionan, al perí́dico de mayor actualidad mundial, las más adecuadas y amplias oficinas modernas. 


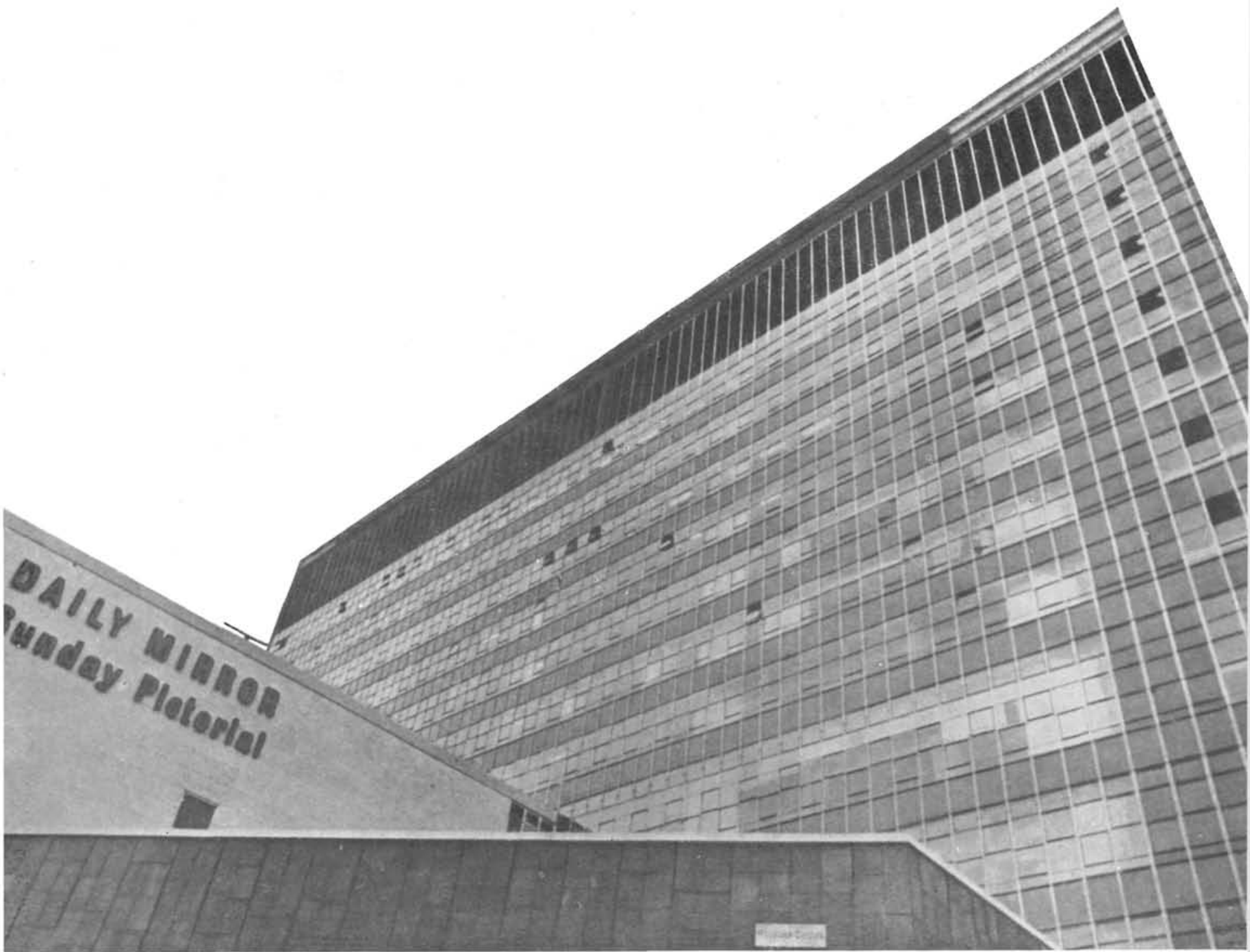

Ha sido construido sobre el terreno que ocupaban antiguamente los almacenes Wallis-destruidos durante la guerra-, emplazado entre el Circus Holborn y las calles New Fetter y Fetter Lane.

Dada la importancia de la situación del solar, y la dimensión del edificio, intervinieron activamente en su proyecto -estudiando detenidamente su forma, medidas, sombra barrida y acabados exteriores-la Corporación de la Ciudad y la Real Comisión de Bellas Artes, junto con otras autoridades.

El edificio tiene 18 plantas; y la alta torre, que se eleva $51,51 \mathrm{~m}$ sobre el nivel de la calle, constituye un grandioso hito de referencia en esta zona de Londres.

Los cuatro sótanos contienen el cuerpo de Prensa. El inferior aloja las máquinas rotativas, cuyas dimensiones son: $49,37 \mathrm{~m}$ de longitud por $7,62 \mathrm{~m}$ de altura, su peso aproximario 800 toneladas y su capacidad de producción, 320.000 pe ródicos a la hora cada una. Y en los espacios circundantes se encuentran: las bobinas de papel-con una capacidad para $1.000 \mathrm{t}-$, fundición de moldes, maquinaria para la instalación de ventilación, depósitos con cabida para 50 t de tinta, otros depósitos de aceite, cintas transportadoras de periódicos y servicios.

La planta baja tiene la entrada principal y el vestíbulo de exposición en la esquina nordeste; la entrada de los empleados está en el suroeste. Una amplia calzada interior, para circulación de vehículos, rodea el departamento de publicación, colocado en el centro del piso. 
plamtas
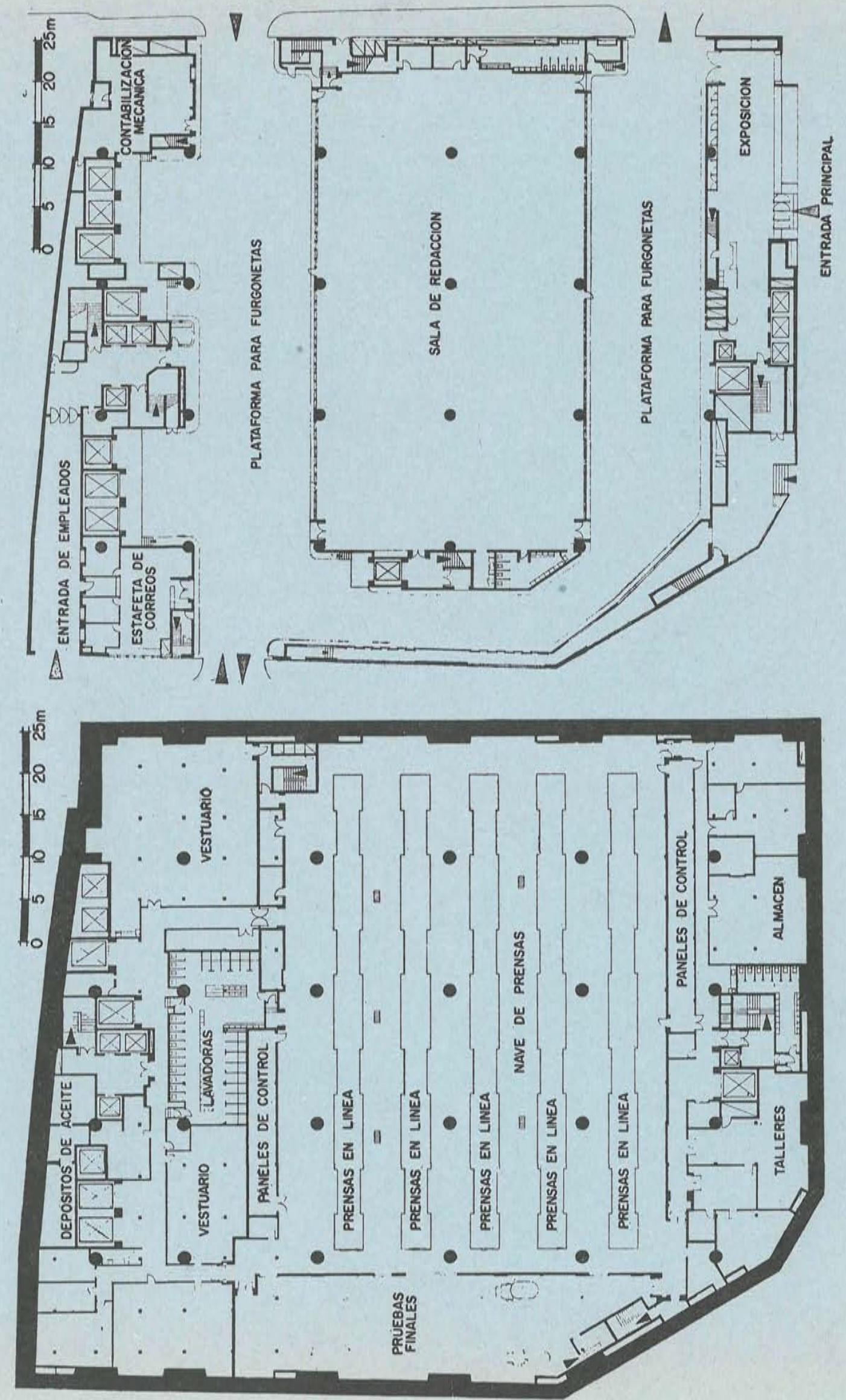


\section{p)a ต}
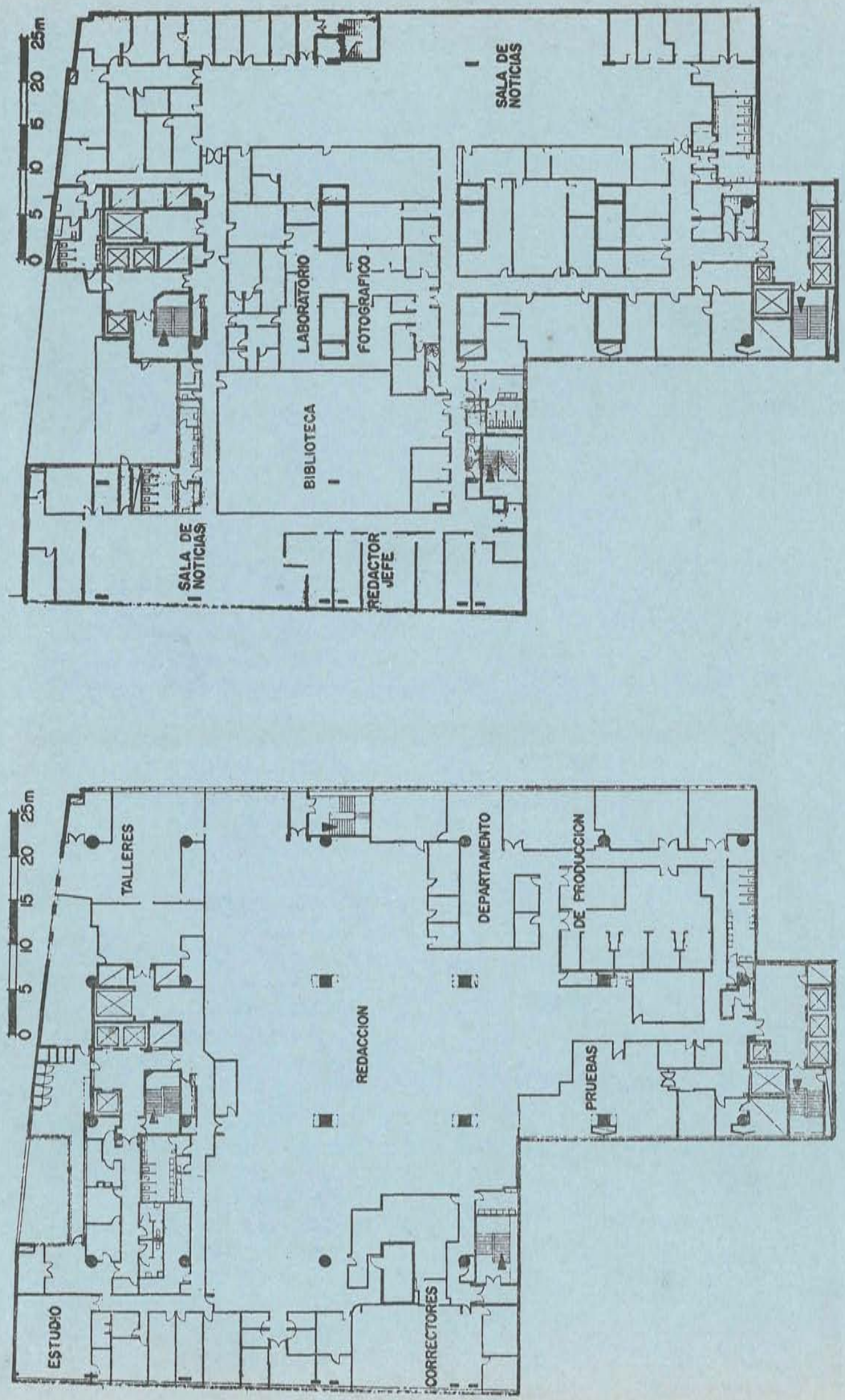

둘 

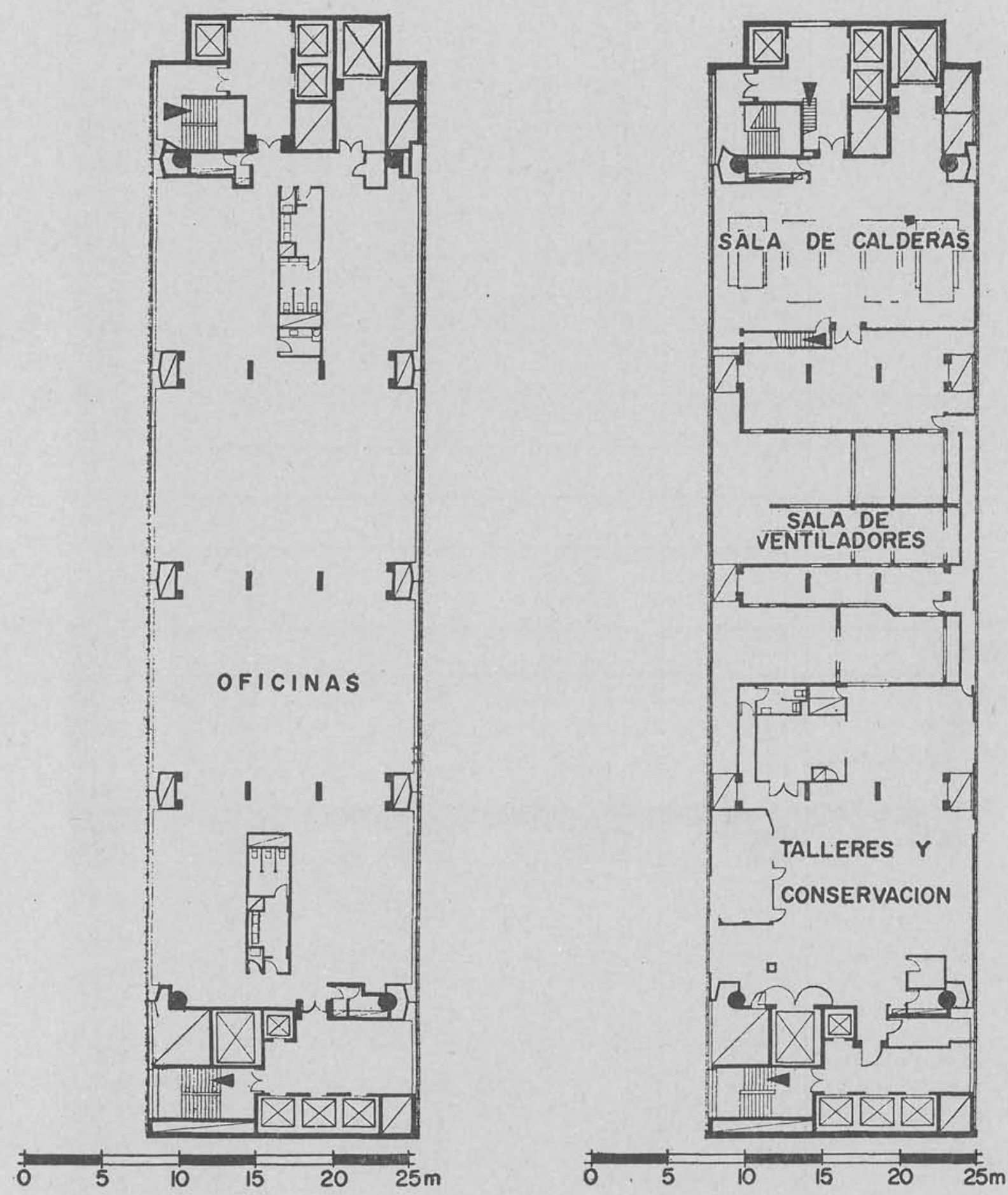

tipo

(1) 


\section{Sección tramsumersal}

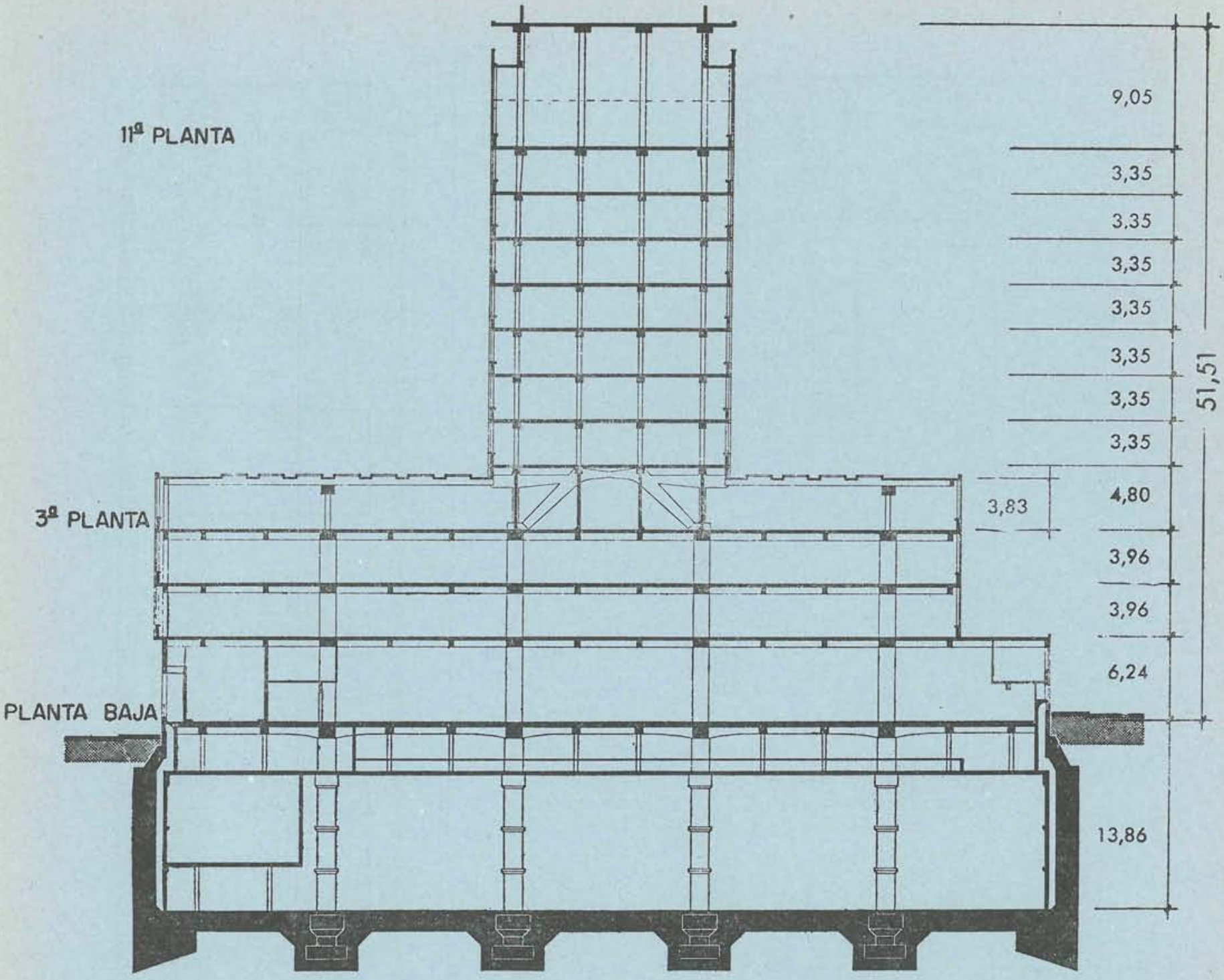

Encima de la planta baja están los entresuelos, ocupados por los servicios contra incendios, oficinas de publicaciones y diversos equipos de trabajo.

La primera planta alberga un gran snack-bar, la parte superior de la zona de publicaciones, y centralita de teléfonos con 98 líneas y 800 extensiones. Los departamentos de producción, composición, grabado, así como un Centro ae asistencia médica están en la segunda planta.

La tercera planta abarca las oficinas principales de la Editorial del "Daily Mirror", "Sunday Pictorial", un departamento de telecomunicación y la biblioteca, que guarda millones de recortes y fotografías de prensa.

La cuarta planta señala el comienzo de la torre, y aquélla, junto con las $5,6,7,8$ y 10, están dedicadas a oficinas. El piso 9 se destina a las oficinas-apartamentos del Director general, y de algunos directores, Sala de Consejo y comedor para jefes.

La planta 11 y su entresuelo están ocupados por la maquinaria de las diversas instalaciones del conjunto edificado. Inmediatamente debajo de la misma ha sido suspendido, con muelles, un cielorraso. Este encierra una gran variedad ae materiales aislantes, con objeto de evitar que los ruidos producidos en el piso superior perturben el trabajo del personal del piso inferior. 

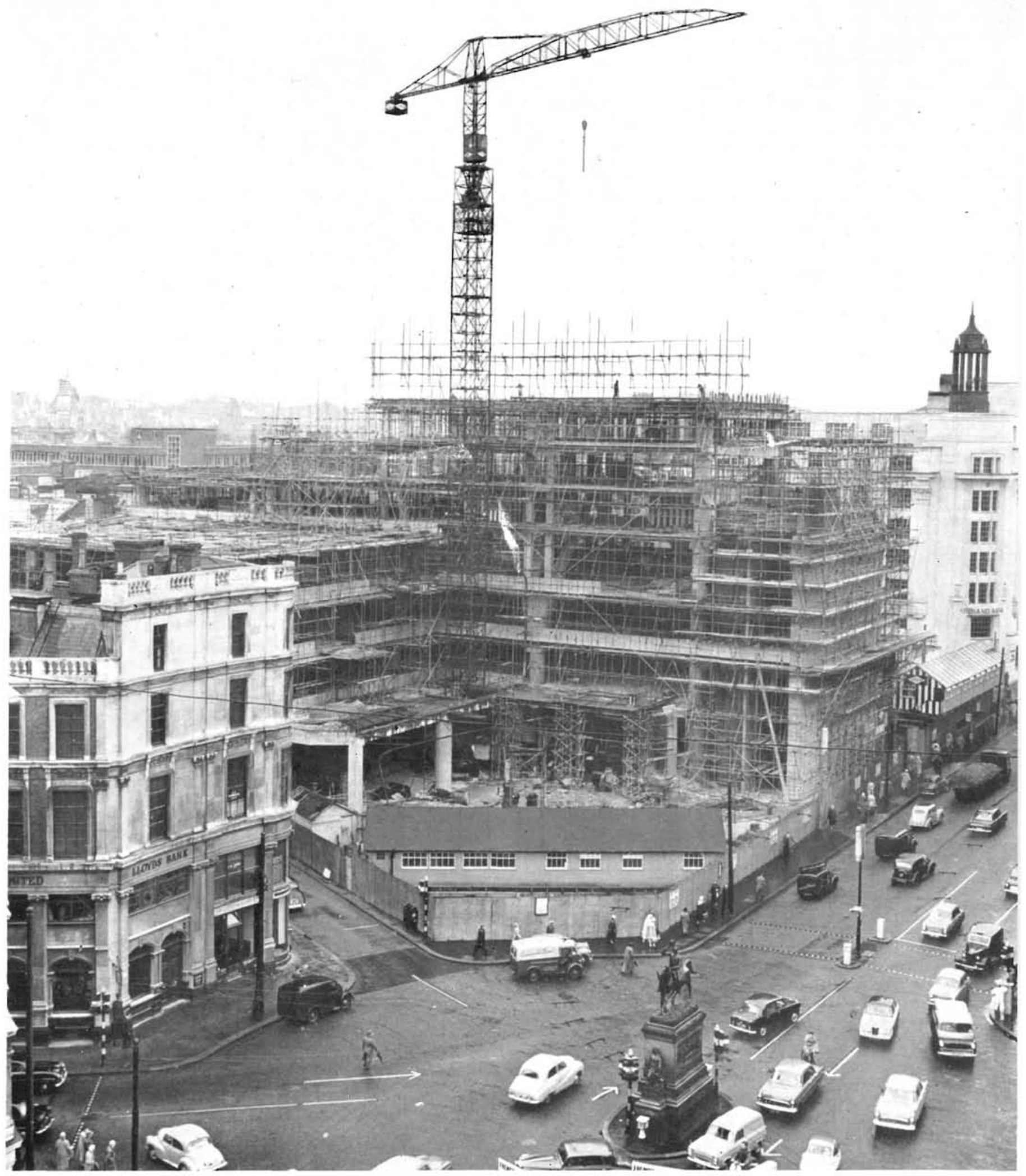

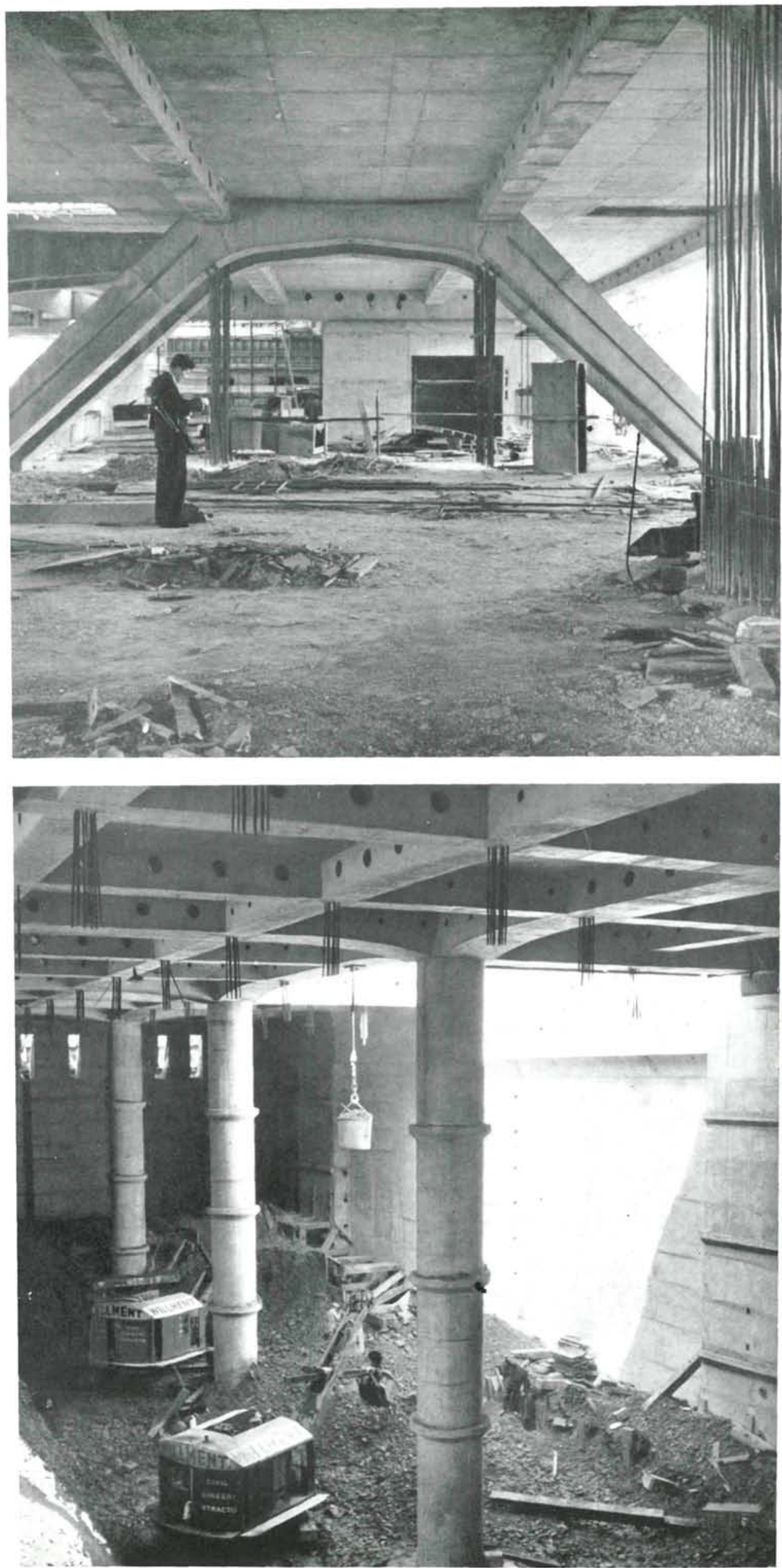

C $\mathbb{0}$ in 5 tै

Todos los pisos de oficinas son calentados mediante convectores situados en los muros perimetrales, controlados termostáticamente, pero con posibilidad de ser regulados manualmente. La ventilación se realiza mecánicamente en los sótanos, planta baja y en los tres primeros pisos.

Para las 3.000 personas empleadas en el edificio hay ascensores que marchan a una velocidad de $213,36 \mathrm{~m}$ por minuto, automáticamente controlados, y que proporcionan un servicio adecuado al número de llamadas y de pasajeros en cada momento. Este sistema reduce el tiempo

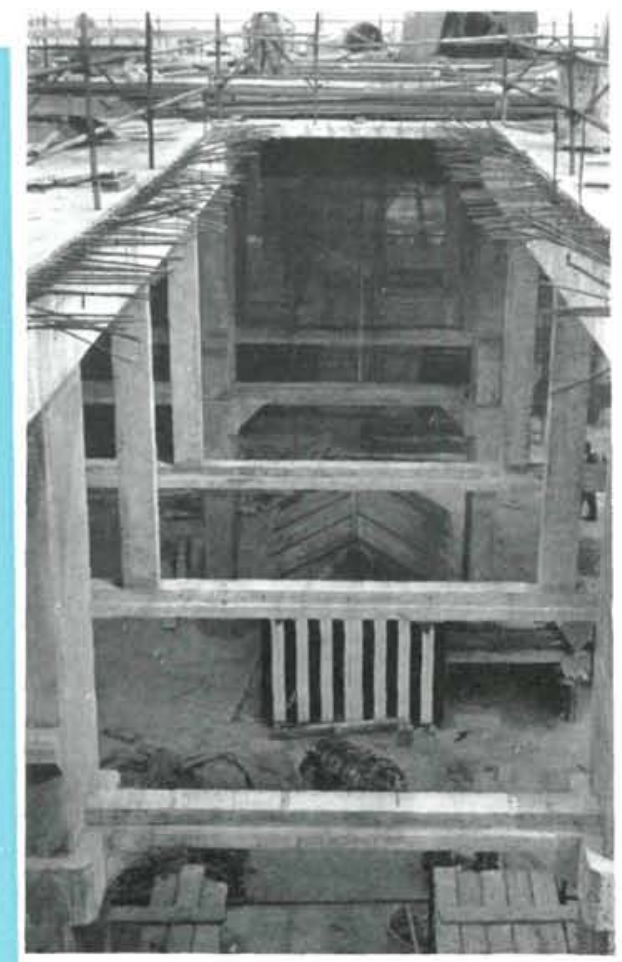

de espera al mismo, pues el ascensor más próximo que viaja en la dirección requerida es parado para recoger una llamada de bajada, registrada en aquella dirección.

Los acabados exteriores del edificio fueron decididos, como dijimos, consuitando con la Real Comisión de Bellas Artes y con destacados diseñadores. 

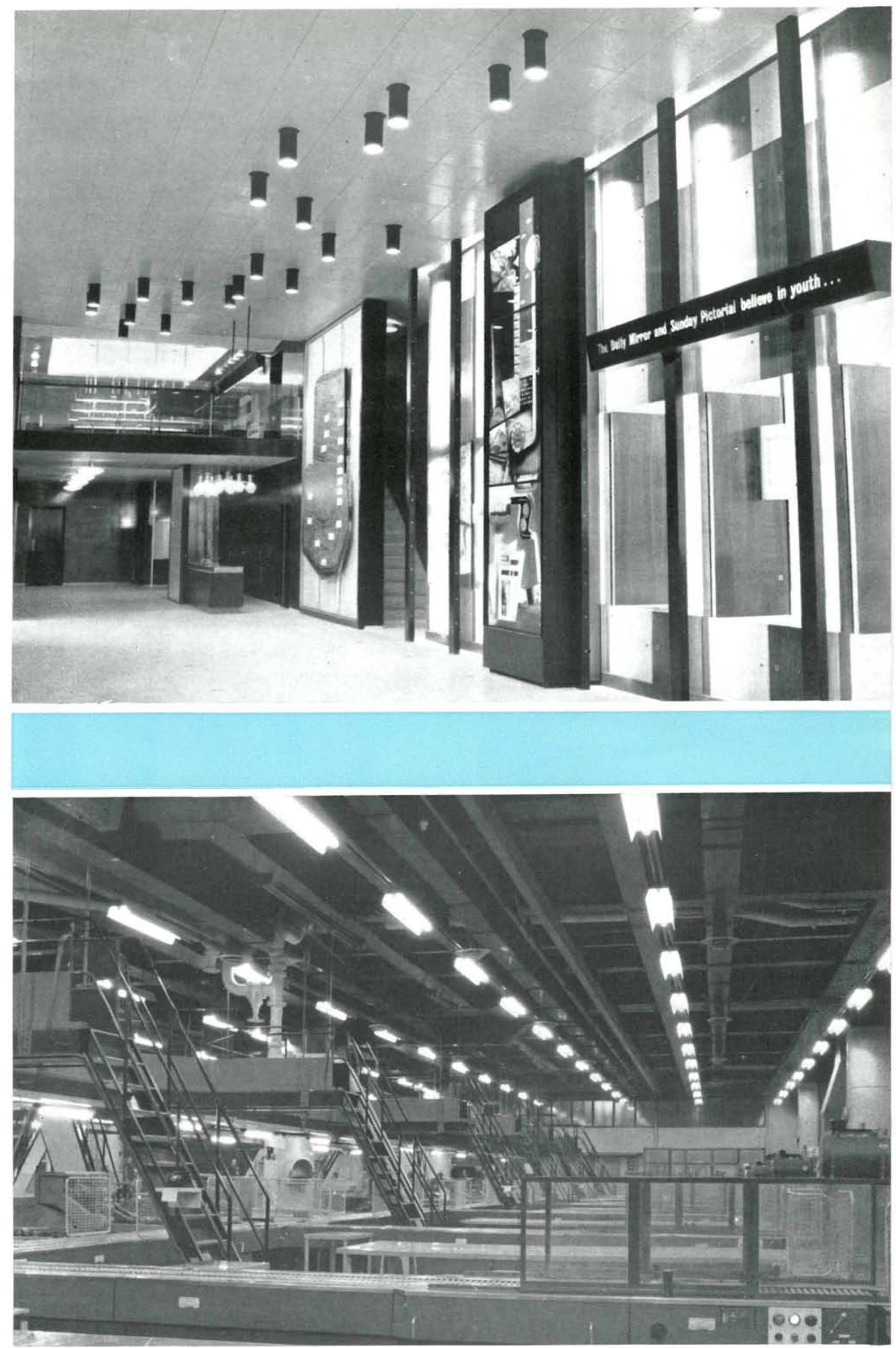

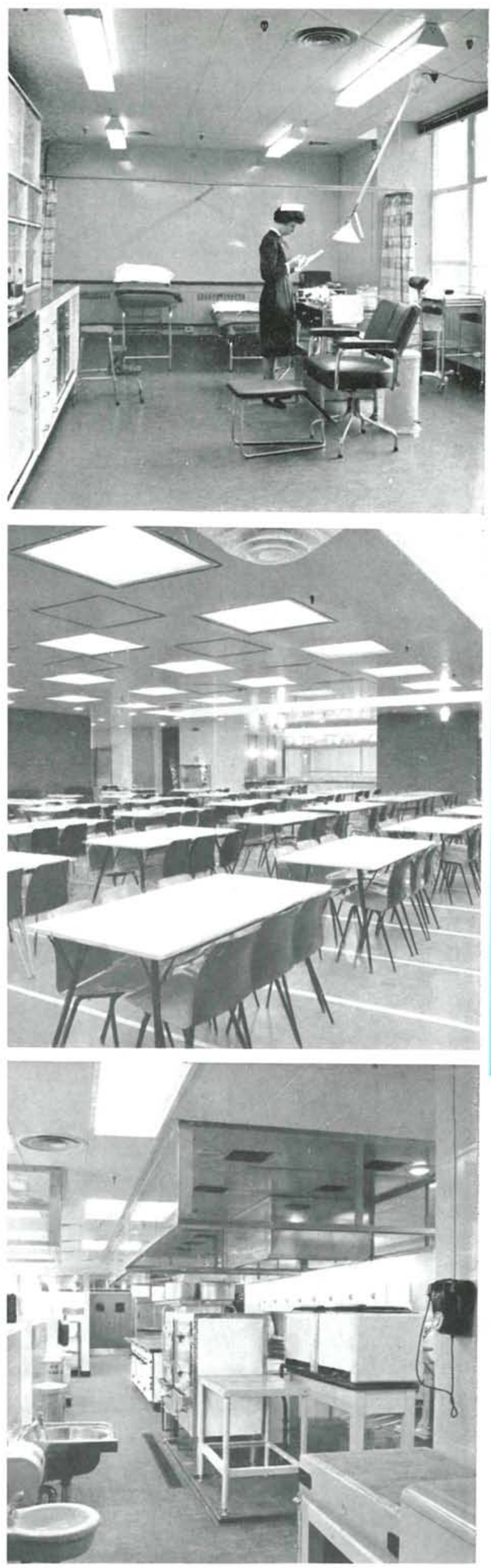

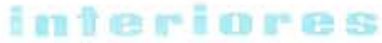

El exterior de la planta baja está revestido con pizarra verde oscura. Sobre éste, en los muros laterales, aparecen, profusamente dispuestos, vidrios grises y entrepaños vitreos-esmaltados, subrayando las ventanas; en contraste con esto, la pared norte va aplicada de mármol blanco.

Por encima de la tercera planta, el bloque de la torre presenta una contraposición de ventanas luminosas y rojos paneles vitreos-esmaltados. La fachada norte, por el contrario, no tiene ventanas y su paramento ofrece unidad de color y textura desde el nivel de la calle a la parte más alta del edificio.

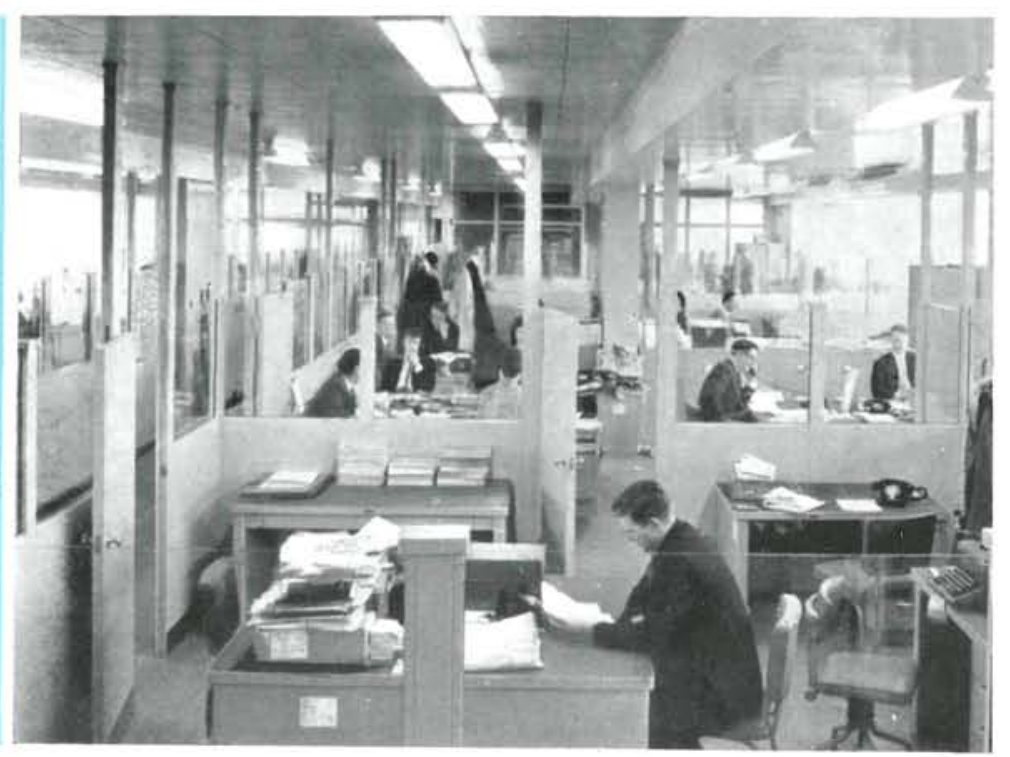

El uso del color ha siao hecho extensivo al interior, en cielorrasos, paredes y pavimentos, habiendo sido utilizados maderas, plásticos, terrazos, gomas y azulejos. Estos últimos no sólo en las zonas de servicios, sino como elementos decorativos.

En definitiva, el "Daily Mirror" se distingue por el acierto $\mathrm{y}$ perfección de sus instalaciones, proporcionando al periódico de información de mayor actualidad, impreso en el mundo, las más adecuadas y extensas oficinas modernas. 


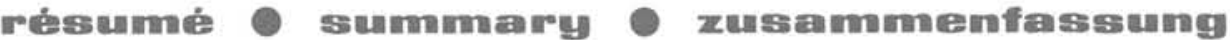

\section{Ediffice du Daily Mimmor - Lomalmess}

Architectes: Sir Owen Williams et collaborateurs.

Anderson Forster et Wilcox. architectes et ingénieurs.

Cet édifice compte 18 étages au total. Les quatre sous-sols sont réservés à la presse. Au rez-de-chaussée sont situés les vestibules d'entrée et une chaussée intérieure pour véhicules. Le premier étage abrite un snack. bar et une centrale téléphonique, le deuxième le département de production et le centre d'assistance médicale et le troisième la bibliothèque et les bureaux principaux.

Le quatrième étage marque le commencement de la partie haute du bloc. Cet étage, ainsi que le 5ème, 6ème, 7ème, sème et le 10ème, sont destinés à des bureaux. Le neuvième étage abrite les bureaux-appartements des directeurs et les salles de Conseil et le onzième l'équipement et les machineries destinés aux diverses installations de l'édifice. Cette haute tour constitue un impressionnant point de repère dans cette zone de Londres.

Le «Daily Mirror» se distingue par le nombre et la perfection de ses installations qui procurent au journal de plus grande actualité mondiale les bureaux les plus amples et les mieux conçus.

\section{"Daig Mirrogo" building, Lomedom}

Sir Owen Williams and collaborators, architects.

Anderson Forster \& Wilcox, engineers.

The building has 18 levels. The Press occupies the 4 basement floors. The ground floor is taken up with the entrance hall, and an indoor carriage way. A snack bar and the telephone operators are situated on the second floor. The production department and the medical services are located on the third storey, whilst the fourth is occupied by the offices and library.

The fifth floor is the beginning of the higher section of the building. This floor and up to including the 11th floor are devoted to office space, except for the 10th storey, which contains the office apart. ments of the directors and the Council Chamber. Equipment related to various services of the building is housed on the 12th storey. Finally, this tall building constitutes a fine landmark in the London skyline.

The Daily Mirror building is outstanding for the appropriate nature, the completeness and the quality of its installations, which thus provide the most widely read paper in the world with outstandingly efficient offices.

\section{Geböiude dies Daily - Mirmmor, Lomedon}

Architekten: Herr Owen Williams und Mitarbeiter.

Anderson Forster und Wilcox, Architekt und Ingenieur.

Das Gebäude verfügt im Ganzen über 18 Stockwerke. Die Presseabteilung ist in den 4 Kellern unterge. bracht. Die Eingangshallen und eine innere Strasse für Fahrzeuge befinden sich im Erdgeschoss. Der erste Stock beherbergt eine Snack - Bar und die Telefon - Zentrale. In dem zweiten Stock finden wir die Produktionsabteilung und das Zentrum der ärztlichen Hilfe, in dem dritten die Hauptbureauräume und die Bibliothek.

Der vierte Stock kennzeichnet den Beginn des hohen Blockes; dieser Stock, sowie der 5., 6., 7., 8. und 10. sind Bureauräumen zugeeignet. Der neunte Stock enthält die Bureauabteilung der Direktoren und Ratssäle und der elfte den Maschinenraum für die einzelnen Anlagen des Gebäudes. Der erhöhte Turm bildet einen grossartigen Markstein in dieser Zone Londons.

Der «Daily Mirror» hebt sich hervor durch den Erfolg, die Anzahl und die Vollkommenheit seiner An. lagen, die der Zeitung mit der grössten Weltaktualität die angemessensten und modernsten grossen Bu. reauräume zur Verfügung stellen. 